\title{
IMPACTO DO CLIMA ORGANIZACIONAL NA SATISFAÇÃO DOS TRABALHADORES DE UMA INSTITUIÇÃO DE ENSINO SUPERIOR
}

IMPACT OF THE ORGANIZATIONAL CLIMATE IN THE SATISFACTION OF WORKERS IN A HIGHER EDUCATION INSTITUTION IMPACTO DEL CLIMA ORGANIZACIONAL EN LA SATISFACCIÓN DE LOS
TRABAJADORES DE UNA INSTITUCIÓN DE ENSEÑANZA SUPERIOR

Amândio Baía (baia@ipg.pt)*

\section{RESUMO}

O Clima Organizacional representa a perceção que os trabalhadores têm da forma como uma empresa oferece as condições adequadas para que eles desenvolvam o seu trabalho da melhor maneira. Um clima organizacional positivo conduz e sustenta a motivação e o desempenho elevado dos trabalhadores. Quando os trabalhadores se sentem motivados, colocam todas as suas capacidades para trabalhar. Esta investigação, sustentada num questionário, apresenta um estudo de caso aos trabalhadores de uma Instituição de Ensino Superior, Instituto Politécnico da Guarda (IPG), com o objetivo de compreender o seu clima organizacional e propor medidas de melhoria. Os resultados apontam para a existência de um clima organizacional ligeiramente positivo no IPG, com forte ênfase na Escola Superior de Turismo e Hotelaria (ESTH), embora os trabalhadores não docentes estejam menos satisfeitos, em geral, do que os trabalhadores docentes. A maioria dos trabalhadores, em todas as unidades orgânicas do IPG, considerou o Sistema de Avaliação de Desempenho não justo e não útil, a remuneração não adequada com as funções desempenhadas, gostariam de ter mais oportunidades de socialização e menos burocracia. Manifestaram ainda o desejo de participar no planeamento dos objetivos de IPG e consideram que têm poucas expectativas de virem a ser promovidos.

Palavras Chave: clima organizacional, gestão de recursos humanos, desempenho, satisfação.

\section{ABSTRACT}

The Organizational Climate represents the perception that workers have of how a company offers the right conditions for them to carry out their work in the best way. A positive organizational climate drives and sustains the workers' motivation and high performance. When workers feel motivated, they put all their skills to work. This research, based on a questionnaire, presents a case study of workers at a Higher Education Institution, Polytechnic Institute of Guarda (IPG), in order to understand their organizational climate and propose improvement measures. The results point out the existence of a slightly positive organizational climate in the IPG, with a strong emphasis on the School of Higher Education in Tourism and Hospitality Management (ESTH), although non-teaching workers are generally less satisfied than professors. Most of the workers in all the organic units of the IPG considered the Performance Evaluation System unjust and not useful, the remuneration not adequate with the functions performed and also they would like to have more opportunities for 
socialization and less bureaucracy. They also expressed the wish to participate in the planning of the IPG's objectives and considered that they had little expectation of being promoted.

Keywords: work climate, human resource management, performance, satisfaction.

\section{RESUMEN}

El clima organizacional representa la percepción que los trabajadores tienen de la forma en que una empresa ofrece las condiciones adecuadas para que ellos desarrollen su trabajo de la mejor manera. Un clima organizacional positivo conduce y sostiene la motivación y el alto rendimiento de los trabajadores. Cuando los trabajadores se sienten motivados, ponen todas sus capacidades para trabajar. Esta investigación, sostenida en un cuestionario, presenta un estudio de caso a los trabajadores de una Institución de Enseñanza Superior, Instituto Politécnico de la Guardia (IPG), con el objetivo de comprender su clima organizacional y proponer medidas de mejora. Los resultados apuntan a la existencia de un clima organizacional ligeramente positivo en el IPG, con fuerte énfasis en la Escuela Superior de Turismo y Hostelería (ESTH), aunque los trabajadores no docentes están menos satisfechos, en general, que los trabajadores docentes. La mayoría de los trabajadores, en todas las unidades orgánicas del IPG, consideró el Sistema de Evaluación de Desempeño no justo y no útil, la remuneración no adecuada con las funciones desempeñadas, quisieran tener más oportunidades de socialización y menos burocracia. Manifestaron el deseo de participar en la planificación de los objetivos de IPG y consideran que tienen pocas expectativas de que se promuevan.

Palabras clave: clima organizacional, gestión de recursos humanos, rendimiento, satisfacción.

\footnotetext{
Guarda - PORTUGAL, UDI - Research Unit for Inland Development of Guarda Polytechnic Institute
}

Submitted: 26th February 2019

Accepted: 13th July 2019 


\section{INTRODUÇÃO}

A avaliação da perceção dos trabalhadores sobre o clima organizacional em que desenvolvem a sua atividade é uma prática comum nas organizações contemporâneas. Este tipo de avaliação per se permite a uma organização diagnosticar e adotar medidas que podem, possivelmente, resultar numa melhoria geral da satisfação dos trabalhadores.

Depois de se avaliar o clima organizacional e aplicar medidas corretivas aos problemas da empresa, pode esperar-se um aumento de produtividade, uma diminuição da taxa de absentismo, uma redução na rotatividade funcional e uma maior satisfação do cliente, entre outros aspetos que tenham impacto nos resultados (Limm et al., 2012).

Quando as pessoas trabalham num ambiente solidário lutam para produzir resultados. Um ambiente deste tipo é chamado de clima organizacional positivo. O clima organizacional é o "clima do local de trabalho". Tal como as condições atmosféricas podem afetar as atividades diárias, o clima organizacional influencia o comportamento no local de trabalho (Galer et al, 2005).

Um bom clima organizacional pode melhorar os hábitos de trabalho individuais, enquanto um clima organizacional pobre pode corroer os bons hábitos de trabalho. Mais importante, um clima organizacional positivo conduz e sustenta a motivação e o desempenho elevado dos trabalhadores (Stringer, 2011). Murphy (2015) refere que "os trabalhadores em ambientes de trabalho positivos superam os que trabalham em climas organizacionais negativos em 10 a 30 por cento".

As instituições de Ensino Superior em Portugal enfrentam grandes desafios - atrair novos alunos, tanto nacionais como internacionais, melhorar a qualidade da sua investigação e aprofundar a sua interação com a sociedade, de forma a financiar os seus orçamentos. Para satisfazer estes desafios precisam de alcançar resultados sustentáveis - fortalecendo a capacidade institucional e melhorando a qualidade dos serviços prestados. Através de um clima organizacional positivo os gestores podem sustentar a motivação e o desempenho dos trabalhadores e melhorar a sua vantagem competitiva.

Esta investigação pretende compreender o clima organizacional de uma instituição pública de ensino superior, Instituto Politécnico da Guarda (IPG) e propor medidas corretivas que fortaleçam e melhorem a sua imagem. Foi escolhida uma amostra aleatória simples dos trabalhadores das diferentes unidades orgânicas do IPG a quem foi administrado um questionário a fim de auscultar a sua satisfação sobre o clima organizacional.

\section{REVISÃO DA LITERATURA}

Independentemente do tipo de organização, todas as empresas precisam de gerir a sua força de trabalho usando políticas e práticas apropriadas. As políticas e práticas precisam de ser coordenadas entre si, assim como com as necessidades da organização. As organizações podem ser mais eficazes se gerirem os seus recursos humanos de forma a entrosarem o número certo de pessoas com os comportamentos apropriados, as competências necessárias e os níveis corretos de motivação exigidos pela organização (Schuler \& Jackson, 2007).

Muitas evidências mostram que trabalhadores e organizações saudáveis andam de mãos dadas. O bem-estar dos trabalhadores tem sido associado ao aumento de vantagens competitivas, produtividade, desempenho, satisfação do cliente e seletividade de 
contratação, bem como à diminuição do absenteísmo, turnover, taxas de lesões e acidentes e custos de saúde (Browne \& Lam, 2008; George \& Jonas (2012); Limm et al., 2011; Noblet \& LaMontagne, 2006; Wright etal., 2007).

O clima organizacional é a atmosfera que prevalece no local de trabalho, em conformidade com o experimentado pelos trabalhadores. O conceito de clima organizacional rendeu muitas definições, descrições e conceitos teóricos.

O clima organizacional foi definido como "o significado compartilhado que os trabalhadores atribuem aos eventos, políticas, práticas e procedimentos que experimentam e aos comportamentos que veem sendo recompensados, apoiados e esperados " (Ehrhart et al., 2014: 69).

O clima organizacional revela "um conjunto de propriedades do ambiente de trabalho, percebido, direta ou indiretamente pelos trabalhadores, que assume ser uma força importante na influência do comportamento dos trabalhadores" (Ivancevich et al., 2014: 554). Esse conceito significa que o clima organizacional é um conjunto de atributos do ambiente de trabalho percebidos pelo trabalhador tanto direta como indiretamente e assume ser uma força importante que afeta o seu comportamento.

Harrison e Shirom citados em Armstrong (2012: 127) afirmam que o clima organizacional se refere às perceções dos membros sobre as características do trabalho, como a tomada de decisão, a liderança e as normas sobre o trabalho.

O clima organizacional é considerado um fator relevante para a gestão, particularmente após mudanças decorrentes dos processos de globalização e internacionalização das organizações (Keller \& Aguiar, 2004). As instituições de ensino superior em Portugal esforçam-se por conseguir a sua internacionalização atraindo estudantes e integrando projetos de investigação de âmbito internacional. Elas perceberam que são organizações empresariais e por isso devem ser competitivas quer localmente quer internacionalmente (Paswan \& Ganesh, 2009).

Tornou-se uma necessidade de todas as organizações empresariais desenvolverem postos de trabalho e um ambiente organizacional persuasivo e efetivo de modo a manter uma força de trabalho motivada, entusiasta e valorizada pelo seu sucesso no trabalho (Malik et al., 2010b).

Uma conceção inicial que parece orientar teorias e pesquisas sobre o constructo do clima organizacional é que os ambientes sociais podem ser caracterizados por um número restrito de dimensões (Patterson et al., 2005). Uma análise detalhada das diferentes escalas de clima organizacional revela uma ampla variedade de dimensões. Por exemplo, os aspetos mais comuns referem-se à autonomia individual, ao nível de estruturação do trabalho, às recompensas, ao apoio e consideração, ao calor humano, à qualidade da liderança, à cooperação da equipa e ao suporte ao cliente, o que indica que as dimensões do clima organizacional irão divergir com o propósito e os critérios de interesse da organização que conduz a investigação (Schneider \& Reichers, 1990).

Para melhorar o clima organizacional, ajuda perceber como o ambiente organizacional afeta as pessoas e como se desenvolve. Um clima organizacional positivo estimula a motivação dos trabalhadores, pois fornece condições sob as quais as pessoas podem perseguir os seus próprios objetivos, enquanto se esforçam para atingir os objetivos de trabalho (Bennis \& Schein, 1966). Todo os trabalhadores têm motivadores - impulsos, necessidades e reservas de energia - que podem levá-los a trabalhar com mais eficiência. Quando os trabalhadores se sentem motivados, querem usar as suas capacidades, na plenitude, no local de trabalho. Eles podem até mesmo fazer esforços que excedam as expectativas do local de trabalho. Muito simplesmente, eles usam todo o seu potencial e isso melhora o seu desempenho no trabalho. 
A motivação dos trabalhadores é o elemento mais importante para que as organizações sejam elas públicas ou privadas, tenham sucesso (Chintallo \& Mahadeo, 2013). Se os trabalhadores estiverem mais motivados, o seu desempenho aumentará (Asim, 2013). A motivação em organizações governamentais e não-governamentais é vital e necessária porque pode mudar e melhorar o desempenho dos trabalhadores de uma forma afirmativa (Aarabi et al., 2013).

Owus e Jones (2012: 157) descobriram que a "motivação do trabalho compreende as forças psicológicas dentro de uma pessoa e determina a direção do comportamento da pessoa numa organização e o nível de esforço e persistência diante dos obstáculos". Smith (2013: 613) descreve a motivação como: "a motivação de trabalho refere-se à força (interna ou externa) que leva os indivíduos a se envolverem com comportamentos intencionais relacionados com o seu trabalho". A motivação no trabalho energiza a pessoa a envolver-se numa tarefa. Os resultados dos estudos de Brown (2011), Shahzad et al. (2014) e Owus (2012) também fornecem evidências de que a motivação no trabalho influencia o desempenho.

O foco no clima organizacional pode aumentar a motivação, o esforço e o empenhamento do trabalhador para o atingir os objetivos da organização, bem como aumentar o seu desempenho (Abdelhadi \& Drach-Zahavy, 2012; Greenslade \& Jimmieson, 2011; Jiang et al., 2015; Neal \& Griffin, 2006; Schneider et al., 2013; Mitonga-Monga et al., 2018).

São os atos dos trabalhadores que agem de maneira consistente com o ambiente de trabalho que, em última instância, produzem os resultados de interesse, como a satisfação do cliente, a redução de acidentes e o desempenho financeiro (Ehrhart \& Raver, 2014; Ostroff et al., 2012).

Como as pesquisas sobre o clima organizacional representam medições da satisfação dos trabalhadores, é importante discutir o conceito de satisfação no trabalho. A satisfação no trabalho é classicamente definida como um estado emocional agradável ou positivo que resulta da avaliação que um funcionário faz sobre a sua experiência de trabalho (Locke, 1976). Representa uma interpretação que envolve as circunstâncias de emprego ou trabalho (salários, promoções, reconhecimento, benefícios, horas de trabalho, condições de trabalho, desempenho, habilidades, conhecimento utilizado) e produz um sentimento de aprovação ou desaprovação, apego ou aversão que constitui a atitude de satisfação ou insatisfação com o trabalho (Corsini, 1999; Muchinsky, 2003). Além disso, considera-se um trabalho satisfatório aquele que oferece a oportunidade de atingir os principais objetivos desejados pelo trabalhador (Tamayo, 2001).

Investigações realizadas por um processo de meta-análise estabeleceram uma relação entre a satisfação dos trabalhadores no trabalho e a qualidade dos serviços prestados aos clientes (Brown \& Lam 2008; Whitman et al., 2010).

Fu et al. (2011) relataram que as facetas da satisfação no trabalho (salário, colegas de trabalho, supervisão e trabalho em si) tiveram um impacto significativo no empenhamento no trabalho. Chen e Liu (2012) descobriram que o desempenho no trabalho foi afetado positivamente pelo envolvimento e satisfação no trabalho e marketing interno. Já Hira e Waqas (2012) descobriram que existe uma relação positiva entre satisfação e desempenho no trabalho numa pesquisa com 335 entrevistados trabalhadores de nível médio do setor bancário. Iqbal et al. (2012) referem que a satisfação no trabalho tem um impacto positivo no desempenho numa pesquisa com 251 respondentes de várias universidades. Vários outros estudos referem que a satisfação no trabalho também teve um impacto significativo no empenhamento sobre o trabalho (Qureshi et al., 2011; Malik et al., 2010a; Samad \& Selangor, 2005).

O desempenho é um tópico amplamente estudado em vários campos, como a administração pública, gestão, psicologia industrial, comportamento de trabalho e gestão de recursos humanos (Andersen et al., 2016; Ritz et al., 2016; Vogel et al., 2016). Um dos conceitos de 
desempenho mais referenciado, o de Campbell citado por Colquit et al. (2015: 32-33), refere que "desempenho no trabalho é o valor do conjunto de comportamentos dos trabalhadores que contribuem, positiva ou negativamente, para o cumprimento das metas de trabalho ".

Edwards e Bell (2008) encontraram uma relação positiva e estatisticamente significativa entre satisfação e desempenho da tarefa. Springer (2011) sugeriu que os gestores podem melhorar o desempenho dos trabalhadores aplicando estratégias de gestão para aumentar a satisfação e a motivação no trabalho.

Landy e Conte (2013: 157) também enfatizam o conceito de desempenho sobre comportamentos relevantes para os objetivos do trabalho "desempenho no trabalho são ações ou comportamentos relevantes para os objetivos da organização medidos em termos de proficiência de cada indivíduo".

Um clima organizacional positivo conduz e sustenta a motivação dos trabalhadores e um desempenho elevado (Goleman, 2001).

\section{METODOLOGIA}

Este estudo pretende compreender e contribuir para a melhoria do clima organizacional de uma Instituição de Ensino Superior Pública em Portugal, o Instituto Politécnico da Guarda (IPG), fundado em 1983. Escolheu-se um estudo de caso, selecionando uma amostra aleatória simples, dos trabalhadores (docentes e não docentes) das Unidades Orgânicas do IPG Escola Superior de Tecnologia.e Gestão (ESTG), Escola Superior de Educação, Comunicação e Desporto (ESECD), Escola Superior de Saúde (ESS), Escola de Turismo e Hotelaria (ESTH), Serviços de Ação Social (SAS) e Serviços Centrais (SC), a quem foi aplicado um questionário.

Schneider e Reichers (1990) referem que "uma análise detalhada das diferentes escalas de clima organizacional revela uma ampla variedade de dimensões que irão divergir com o propósito e os critérios de interesse da organização que conduz a investigação" Levando em conta os aspetos que podem influenciar o clima organizacional e a revisão da literatura, três dimensões foram consideradas neste estudo: Organização do Trabalho, Realização do Trabalho e Condições de Trabalho. A confiabilidade dessas dimensões foi calculada usando o coeficiente Alfa de Cronbach.

O questionário, constituído por questões fechadas, foi estruturado em duas partes. Na primeira parte, foram recolhidos dados sobre as caraterísticas dos respondentes: unidade orgânica, atividade, género, idade, estado civil, número de filhos, tempo de serviço no IPG (tenure) e categoria profissional.

Na segunda parte, foram recolhidos dados sobre a perceção que os respondentes tinham sobre as três dimensões, materializados numa escala de satisfação de Likert com níveis de 1 a 5, em que 1 representava "nada" e 5 "muito".

Para garantir a validade do questionário, realizou-se um pré-teste para um grupo de 10 pessoas e solicitou-se o feedback da coerência e compreensão global, a fim de realizar os ajustes necessários.

Com o intuito de melhorar o clima organizacional do IPG, as respostas dos itens e dimensões foram classificadas de verde se a sua média $>4$, de amarelo se $3 \leq$ média $\leq 4$ e de vermelho se média<3. Os itens classificados como vermelho precisam de medidas de ação especiais e intervenção imediata para serem melhorados. Os itens classificados como amarelos precisam de atenção para melhorar e evoluir para o verde. Os itens classificados como verde precisam 
de ser estimulados para serem mantidos e melhorados. Considerou-se como positivo um grau médio de satisfação superior a 3,00.

A fim de serem definidas e implementadas medidas específicas de melhoria, suportadas nas respostas dos respondentes, foram testadas as seguintes hipóteses:

$H_{\mathbf{i}}$ - Não existe diferença entre o grau de satisfação dos itens e das dimensões do Clima Organizacional relativamente ao fator i ( $i=$ unidade orgânica, género, idade, qualificações dos trabalhadores, tenure, tipo de categoria profissional).

Para avaliar as diferenças médias das variáveis contextuais relacionadas com o clima organizacional do IPG e testar as hipóteses utilizou-se a ANOVA unidirecional, o teste $U$ de Mann-Whitney e o teste não-paramétrico de Kruskal-Wallis, seguido de comparações múltiplas das médias da ordem com a ANOVA Tukey, conforme descrito por Maroco (2014). A análise de regressão stepwise foi usada para encontrar os itens relacionados com os itens classificados de vermelho, de modo a que as intervenções possam aumentar a sua eficiência.

A população do estudo é composta por 338 trabalhadores (181 docentes e 157 não docentes), que exercem diretamente funções nas diversas unidades orgânicas do IPG, aos quais foi aplicado o questionário. A recolha de dados ocorreu entre os dias 01 e 30 de Junho de 2018 , tendo sido recolhidos 171 questionários válidos, representando 50,5\% da população.

A Tabela 1 apresenta o número de trabalhadores que exercem funções em cada unidade orgânica e o número de questionários válidos recolhidos.

Tabela 1 - Comparação entre os trabalhadores da população e da amostra

\begin{tabular}{lcccc}
\cline { 2 - 5 } & \multicolumn{2}{c}{ População } & \multicolumn{2}{c}{ Amostra } \\
\cline { 2 - 5 } & Frequência & \% & Frequência & $\%$ \\
\hline ESTG & 107 & 31,7 & 49 & 28,7 \\
\hline ESECD & 60 & 17,8 & 30 & 17,5 \\
\hline ESS & 29 & 8,6 & 12 & 7,0 \\
\hline ESTH & 26 & 7,7 & 21 & 12,3 \\
\hline SAS & 45 & 13,3 & 15 & 8,8 \\
\hline SC & 71 & 21,0 & 44 & 25,7 \\
\hline
\end{tabular}

Foi definido um nível de significância de 5\% e um erro amostral de 4,44\%.Todo o trabalho estatístico foi realizado no software IBM SPSS (v25 - SPSS Inc. Chicago, IL) e Excel (Microsoft Office 2015, Microsoft Corporation).

\section{ANÁLISE E DISCUSSÃO DOS RESULTADOS}

A amostra é composta principalmente por indivíduos do sexo feminino $(56,7 \%)$. A maior parte dos respondentes tem uma idade entre os 41 a 60 anos ( $88,3 \%$ ) sendo que $79,5 \%$ são casados e $69,6 \%$ têm 1 ou 2 filhos (Figura 1). 


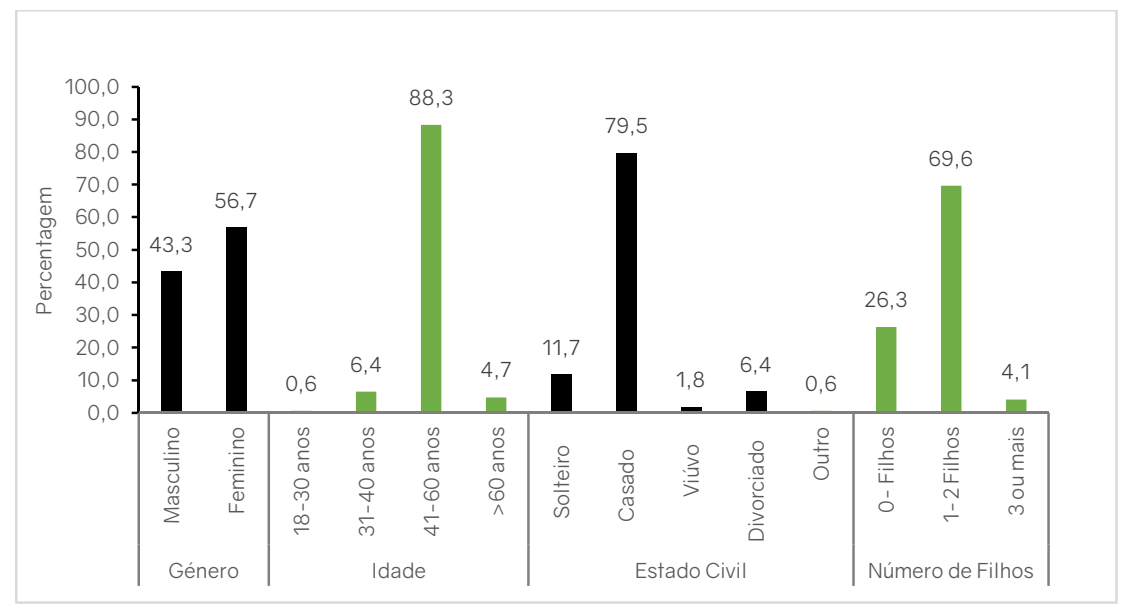

Figura 1 - Género, Idade, Estado Civil e Número de Filhos

O IPG apresenta duas categorias profissionais distintas: 'docentes' e 'não docentes'. A amostra é composta por $46,8 \%$ de pessoal não docente e $53,2 \%$ de docente, $28,7 \%$ dos entrevistados são de ESTG, 25,7\% dos SC e 56,1\% trabalham no IPG há mais de 20 anos (Figura 2).

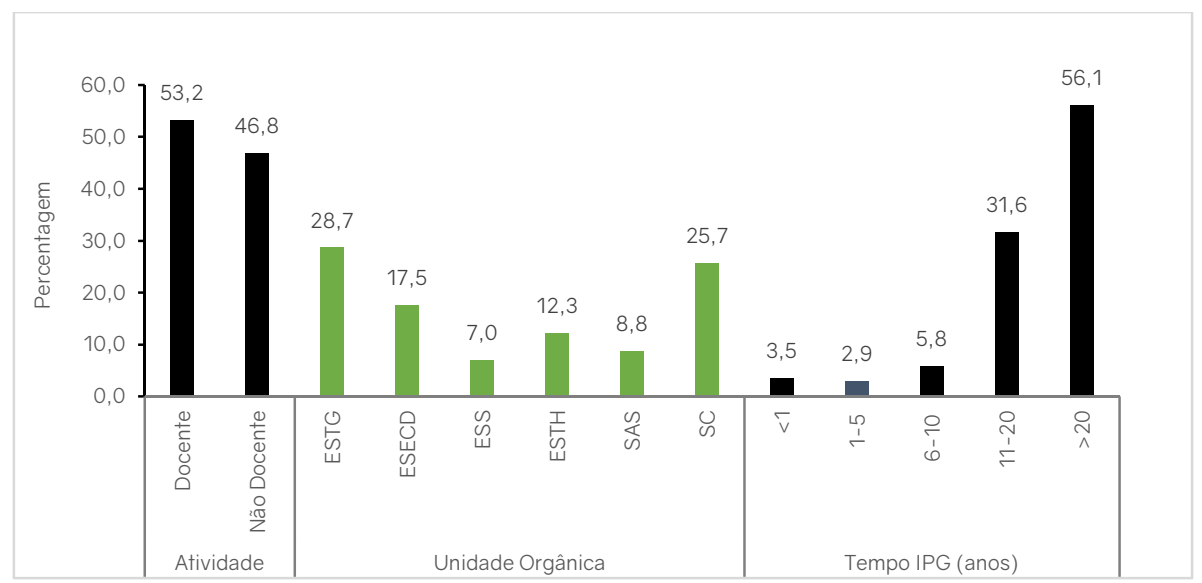

Figura 2 - Atividade, Unidade Orgânica e Tempo de Serviço (IPG)

Quanto às habilitações literárias, a amostra é constituída, maioritariamente, por doutores $(34,5 \%)$ e por mestres (33,9\%), sendo que muitos deles são docentes nas unidades orgânicas, e a maioria detém a categoria profissional de Professor Adjunto ou Equiparado. (Figura 3). 


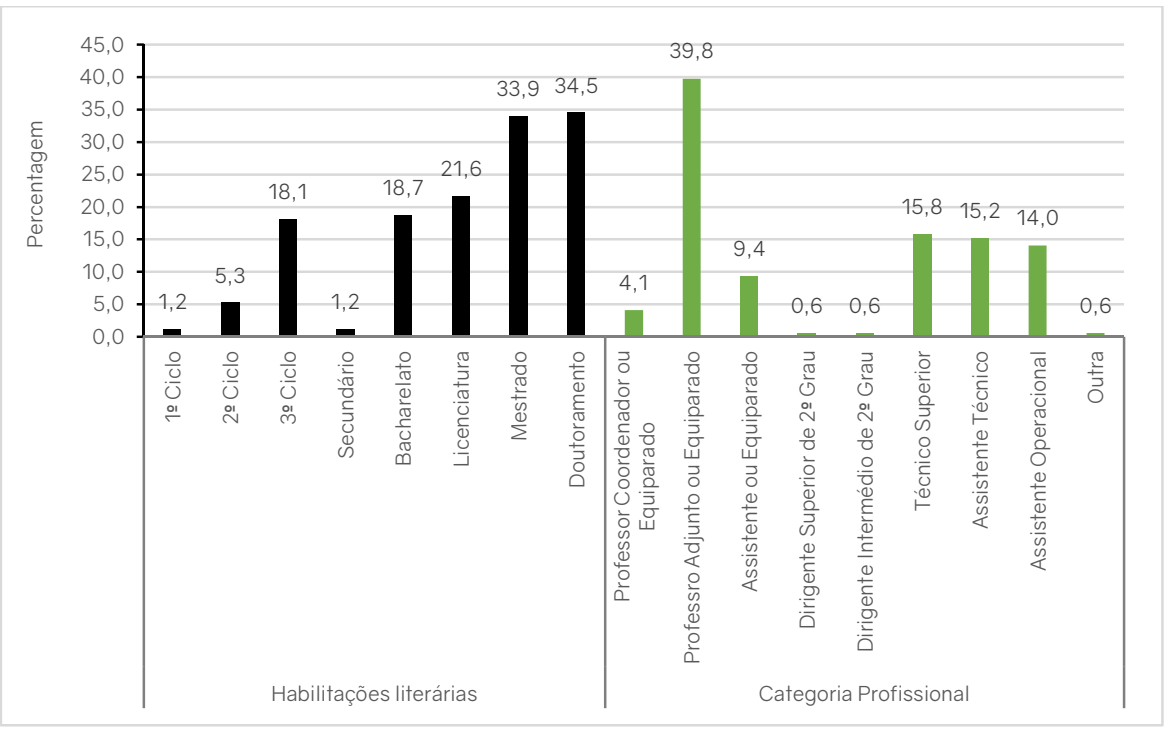

Figura 3 - Habilitações Literárias e Categoria Profissional

A comparação da amostra recolhida nas diferentes unidades orgânicas do IPG com a população dessas unidades orgânicas (Tabela 1) mostra a ausência de diferenças significativas entre elas, concluindo-se que a amostra recolhida e a população são homogêneas [Chi-Square $=10,133(p>0,05)$ ].

Utilizou-se o coeficiente Alfa de Cronbach para calcular os coeficientes de consistência interna dos itens incluídos no questionário. A dimensão Organização do Trabalho consiste de 12 itens ( $\alpha=0,889)$, a dimensão Realização do Trabalho, consiste de 9 itens $(\alpha=0,848)$, e a dimensão Condições de Trabalho, consiste de 11 itens $(\alpha=0,801)$. Os resultados da análise de confiabilidade mostraram que os itens nas três dimensões tinham elevado poder discriminatório (Mallery, 2016).

Foi potenciado para 0,842 o coeficiente Alfa de Cronbach da dimensão Condições de Trabalho com a exclusão do item Sofreu algum tipo de discriminação nos últimos 2 anos (raça, religião, sexo, assédio moral, etc.) (Tabela 2).

Tabela 2 - Alfa de Cronbach se o Item for apagado

\begin{tabular}{lcccc}
\cline { 2 - 4 } & $\begin{array}{c}\text { Média da Escala } \\
\text { se o Item for } \\
\text { Apagado }\end{array}$ & $\begin{array}{c}\text { Variação da } \\
\text { Escala se o Item } \\
\text { for Apagado }\end{array}$ & $\begin{array}{c}\text { Item corrigido- } \\
\text { Correlação Total }\end{array}$ & $\begin{array}{c}\text { Alfa de Cronbach } \\
\text { se o Item for } \\
\text { Apagado }\end{array}$ \\
\hline $\begin{array}{l}\text { Sofreu algum tipo de discriminação } \\
\text { nos últimos 2 anos (raça, religião, } \\
\text { sexo, assédio moral, etc.) }\end{array}$ & 31,2632 & 42,442 & $-0,078$ & 0,842 \\
\hline
\end{tabular}

A maioria dos itens $(83,3 \%)$ pertencentes à dimensão Organização do Trabalho tiveram uma classificação amarela. Os itens: Os processos são fluidos (pouca papelada)e Oportunidade de participação nos objetivos de planeamento do IPG, tiveram uma classificação vermelha, o que significa que precisam de medidas especiais de ação imediatas. Nenhum dos itens foi classificado como verde (Tabela 3). 


\begin{tabular}{|c|c|c|c|c|c|c|c|}
\hline \multicolumn{8}{|c|}{ Organização do Trabalho } \\
\hline Itens & 1 & 2 & 3 & 4 & 5 & Média & $\begin{array}{l}\text { Desvio } \\
\text { Padrão }\end{array}$ \\
\hline $\begin{array}{l}\text { Confiança na capacidade de liderança dos } \\
\text { superiores hierárquicos }\end{array}$ & 4,7 & 6,4 & 24,6 & 40,4 & 24,0 & 3,73 & 1,046 \\
\hline $\begin{array}{l}\text { A informação recebida é adequada (precisa, } \\
\text { atempada, clara e útil) }\end{array}$ & 5,3 & 11,7 & 33,9 & 38,0 & 11,1 & 3,38 & 1,007 \\
\hline Sente que é tratado com respeito e civismo & 4,7 & 5,3 & 21,1 & 44,4 & 24,6 & 3,79 & 1,024 \\
\hline $\begin{array}{l}\text { E fomentada uma cultura de Inovação } \\
\text { (aceitação de novas ideias e processos) }\end{array}$ & 2,9 & 15,8 & 37,4 & 33,3 & 10,5 & 3,33 & 0,963 \\
\hline Os processos são fluidos (pouca papelada) & 25,7 & 31,6 & 31,6 & 7,6 & 3,5 & 2,32 & 1,049 \\
\hline $\begin{array}{l}\text { Grau de interatividade entre o IPG e a } \\
\text { Comunidade em geral }\end{array}$ & 2,3 & 10,5 & 40,9 & 36,8 & 9,4 & 3,40 & 0,885 \\
\hline Conhece os objetivos do IPG & 0,6 & 8,2 & 25,1 & 47,4 & 18,7 & 3,75 & 0,873 \\
\hline $\begin{array}{l}\text { Perceção da qualidade da imagem que o IPG } \\
\text { tem perante a sociedade }\end{array}$ & 1,8 & 7,0 & 39,2 & 39,8 & 12,3 & 3,54 & 0,863 \\
\hline $\begin{array}{l}\text { Oportunidade de participação nos objetivos } \\
\text { de planeamento do IPG }\end{array}$ & 11,1 & 24,6 & 39,2 & 19,9 & 5,3 & 2,84 & 1,039 \\
\hline Sente-se orgulhoso em trabalhar no IPG & 1,8 & 4,1 & 21,6 & 39,8 & 32,7 & 3,98 & 0,933 \\
\hline $\begin{array}{l}\text { Pensa que os superiores hierárquicos agem } \\
\text { eticamente nas suas decisões }\end{array}$ & 5,8 & 11,1 & 31,6 & 36,3 & 15,2 & 3,44 & 1,063 \\
\hline $\begin{array}{l}\text { Grau de Comunicação Interna - (os estatutos, } \\
\text { regimentos e manuais de atividades, estão } \\
\text { bem definidos e conhecidos por todos e os } \\
\text { eventos divulgados atempadamente) }\end{array}$ & 4,7 & 14,0 & 39,2 & 33,9 & 8,2 & 3,27 & 0,963 \\
\hline
\end{tabular}

Sete itens $(77,8 \%)$ da dimensão Realização do Trabalhoforam classificados como amarelos e dois $(22,2 \%)$ como vermelhos (Tabela 4). Esses dois itens: Oportunidade de ser promovido e A remuneração é adequada com as funções desempenhadas representaram questões especiais que podem inibir a motivação (Robbins \& Judge, 2019).

Tabela 4 - Dimensão - Realização do Trabalho

\begin{tabular}{|c|c|c|c|c|c|c|c|}
\hline \multicolumn{8}{|c|}{ Realização do Trabalho } \\
\hline Itens & 1 & 2 & 3 & 4 & 5 & Média & $\begin{array}{l}\text { Desvio } \\
\text { Padrão }\end{array}$ \\
\hline Oportunidade de ser promovido & 27,5 & 20,5 & 32,2 & 14,0 & 5,8 & 2,50 & 1,200 \\
\hline $\begin{array}{l}\text { Grau de autonomia para tomar decisões no } \\
\text { trabalho }\end{array}$ & 6,4 & 14,0 & 33,9 & 36,8 & 8,8 & 3,27 & 1,023 \\
\hline $\begin{array}{l}\text { Participação nas decisões em especial nas } \\
\text { que o afetam }\end{array}$ & 5,3 & 17,5 & 40,9 & 28,1 & 8,2 & 3,16 & 0,986 \\
\hline Grau de Comunicação com os superiores & 3,5 & 8,8 & 25,7 & 42,1 & 19,9 & 3,66 & 1,007 \\
\hline $\begin{array}{l}\text { Relacionamento entre os colegas de } \\
\text { trabalho }\end{array}$ & 1,2 & 2,3 & 26,3 & 48,5 & 21,6 & 3,87 & 0,816 \\
\hline $\begin{array}{l}\text { A distribuição do trabalho é feito de forma } \\
\text { justa (equitativa) }\end{array}$ & 10,5 & 18,1 & 37,4 & 24,0 & 9,9 & 3,05 & 1,116 \\
\hline $\begin{array}{l}\text { A remuneração é adequada com as funções } \\
\text { desempenhadas }\end{array}$ & 17,5 & 15,8 & 38,6 & 23,4 & 4,7 & 2,82 & 1,120 \\
\hline $\begin{array}{l}\vec{E} \text { proporcionada formação suficiente e } \\
\text { adequada para as funções que desempenha }\end{array}$ & 9,4 & 18,7 & 39,8 & 22,2 & 9,9 & 3,05 & 1,089 \\
\hline É feliz no seu local de trabalho? & 5,3 & 9,4 & 26,3 & 40,4 & 18,7 & 3,58 & 1,062 \\
\hline
\end{tabular}

Três itens da dimensão Condições de Trabalho tiveram classificação de vermelho: Considera útil e justo o Sistema de Avaliação de Desempenho, São fomentadas oportunidades de socialização (festas, piqueniques, passeios, etc.) e Acha o seu trabalho repetitivo. Apenas o item relacionado com a discriminação teve uma classificação de verde (Tabela 5).

Tabela 5 - Dimensão - Condições do Trabalho 


\begin{tabular}{|c|c|c|c|c|c|c|c|}
\hline \multicolumn{8}{|c|}{ Condições do Trabalho } \\
\hline Itens & 1 & 2 & 3 & 4 & 5 & Média & $\begin{array}{l}\text { Desvio } \\
\text { Padrão }\end{array}$ \\
\hline Qualidade geral da supervisão & 5,3 & 8,8 & 46,2 & 32,7 & 7,0 & 3,27 & 0,914 \\
\hline $\begin{array}{l}\text { Sente que o seu trabalho é valorizado na } \\
\text { Instituição }\end{array}$ & 8,2 & 17,0 & 40,4 & 24,6 & 9,9 & 3,11 & 1,065 \\
\hline $\begin{array}{l}\text { Conciliação das exigências do trabalho com } \\
\text { a sua vida pessoal }\end{array}$ & 4,7 & 7,6 & 42,7 & 36,3 & 8,8 & 3,37 & 0,920 \\
\hline $\begin{array}{l}\text { Reconhecimento do seu trabalho pelos } \\
\text { superiores }\end{array}$ & 7,6 & 14,6 & 39,2 & 28,7 & 9,9 & 3,19 & 1,052 \\
\hline Adequação do Horário de Trabalho & 4,7 & 6,4 & 30,4 & 43,3 & 15,2 & 3,58 & 0,981 \\
\hline $\begin{array}{l}\text { Sofreu algum tipo de discriminação nos } \\
\text { últimos } 2 \text { anos (raça, religião, sexo, assédio } \\
\text { moral, etc.) }\end{array}$ & 7,6 & 6,4 & 5,8 & 7,0 & 73,1 & 4,32 & 1,281 \\
\hline $\begin{array}{l}\text { O ambiente de trabalho favorece a execução } \\
\text { das suas atividades }\end{array}$ & 4,1 & 8,8 & 40,9 & 38,0 & 8,2 & 3,37 & 0,908 \\
\hline $\begin{array}{l}\text { Considera útil e justo o Sistema de Avaliação } \\
\text { de Desempenho }\end{array}$ & 29,8 & 25,7 & 32,2 & 6,4 & 5,8 & 2,33 & 1,142 \\
\hline $\begin{array}{l}\text { São fomentadas oportunidades de } \\
\text { socialização (festas, piqueniques, passeios, } \\
\text { etc.) }\end{array}$ & 12,9 & 27,5 & 39,8 & 13,5 & 6,4 & 2,73 & 1,056 \\
\hline Acha o seu trabalho não repetitivo & 10,5 & 19,9 & 36,3 & 28,1 & 5,3 & 2,98 & 1,057 \\
\hline $\begin{array}{l}\text { As condições físicas do seu trabalho são } \\
\text { satisfatórias (Ruído, Higiene, Mobiliário, } \\
\text { Equipamentos, Gabinetes) para desenvolver } \\
\text { as suas atividades }\end{array}$ & 5,3 & 14,0 & 33,3 & 36,8 & 10,5 & 3,33 & 1,017 \\
\hline
\end{tabular}

Podemos ver na Tabela 6 que, embora os resultados da amostra mostrem que o clima organizacional no IPG é ligeiramente positivo (média=3,29), ainda existe uma grande oportunidade de melhoria. Todas as médias das dimensões se enquadram na classificação amarela, mas a dimensão Realização do Trabalho apresenta a menor média $(3,22)$ significando que existe uma oportunidade para a gestão do IPG aumentar o desempenho (Gong et al., 2009; Greenberg, 2011a; Iqbal et al., 2012, Judge \& Church, 2000; Waqas, 2012). Os trabalhadores da ESTH são os mais satisfeitos e os da ESTG são os menos satisfeitos. Vinte e quatro dos itens foram classificados de amarelo, sete de vermelho e apenas um dos itens, dos trinta e dois itens, mereceu a classificação de verde.

Tabela 6 - Médias e desvios padrões do Clima Organizacional por fator e dimensões

\begin{tabular}{lcc}
\hline & Média & Desvio Padrão \\
\hline Clima Organizacional & 3,29 & 0,590 \\
\hline Dimensões & 3,40 & \\
\hline Organização do trabalho & 0,643 \\
\hline Realização do trabalho & 3,22 & 0,707 \\
\hline Condições de Trabalho & 3,23 & 0,595 \\
\hline Unidade Orgânica & \\
\hline ESTG & 3,21 & 0,572 \\
\hline ESECD & 3,33 & 0,550 \\
\hline ESS & 3,27 & 0,648 \\
\hline ESTH & 3,59 & 0,566 \\
\hline SAS & 3,26 & 0,589 \\
\hline CS & 3,24 & 0,608 \\
\hline Atividade & \\
\hline Docente & 3,32 & 0,613 \\
\hline Não docente & 3,27 & 0,559 \\
\hline
\end{tabular}

Utilizou-se a correlação de Spearman para determinar a relação entre as dimensões. Verificou-se uma correlação positiva forte entre todas as dimensões, sendo que todas são estatisticamente significativas. (Tabela 7). 
Tabela 7 - Coeficiente de Correlação de Spearman

\begin{tabular}{lccc}
\hline \multicolumn{1}{c}{ Dimensão } & $\mathbf{1}$ & $\mathbf{2}$ & $\mathbf{3}$ \\
\hline Organização do Trabalho & - & & \\
\hline Realização do Trabalho & $0,749^{*}$ & - & - \\
\hline Condições de Trabalho & $0,716^{* *}$ & $0,743^{* *}$ & \\
\hline${ }^{* *}$. A correlação é significativa no nível 0,01 (2 extremidades).
\end{tabular}

A análise de regressão stepwise foi usada para determinar os itens que apresentam a maior relação com os sete itens classificados de vermelho, de modo a que as intervenções melhorem o seu grau de satisfação.

Os processos são fluidos (pouca papelada) $\left(R^{2}=0,035\right)$

- As condições físicas do seu trabalho são satisfatórias (Ruído, Higiene, Mobiliário, Equipamentos, Gabinetes) para desenvolver as suas atividades $(\beta=-0,187, p<0,014)$

Oportunidade de participação nos objetivos de planeamento do IPG $\left(R^{2}=0,411\right)$

- Grau de interatividade entre o IPG e a Comunidade em geral $(\beta=0,298, p<0,000)$

- Participação nas decisões em especial nas que o afetam $(\beta=0,256, p<0,000)$

- Sente-se orgulhoso em trabalhar no IPG $(\beta=0,215, p<0,003)$

- Adequação do Horário de Trabalho $(\beta=0,129, p<0,003)$

Oportunidade de ser promovido $\left(R^{2}=0.464\right)$

- Grau de autonomia para tomar decisões no trabalho $(\beta=0,345, p<0,000)$

- A remuneração é adequada com as funções desempenhadas $(\beta=0,318, p<0,000)$

- Sente que o seu trabalho é valorizado na Instituição $(\beta=0,228, p<0,006)$

- O ambiente de trabalho favorece a execução das suas atividades $(\beta=-0,261, p<0,001)$

- Qualidade geral da supervisão $(\beta=-0,180, p<0,035)$

A remuneração é adequada com as funções desempenhadas $\left(R^{2}=0,471\right)$

- É proporcionada formação suficiente e adequada para as funções que desempenha $(\beta=0,375, p<0,000)$

- Oportunidade de ser promovido $(\beta=0,382, p<0,000)$

- Considera útil e justo o Sistema de Avaliação de Desempenho ( $\beta=0,161, p<0,019)$

- Sente que o seu trabalho é valorizado na Instituição $(\beta=-0,222, p<0,005)$

- Grau de Comunicação Interna - (os estatutos, regimentos e manuais de atividades, estão bem definidos e conhecidos por todos e os eventos divulgados atempadamente) $(\beta=0,173, p<0,021)$

- Perceção da qualidade da imagem que o IPG tem perante a sociedade $(\beta=-0,164$, $p<0,015)$

- A distribuição do trabalho é feito de forma justa (equitativa) $(\beta=0,151, p<0,027)$

Considera útil e justo o Sistema de Avaliação de Desempenho $\left(R^{2}=0,362\right)$

- É feliz no seu local de trabalho? $(\beta=0,253, p<0,001)$

- São fomentadas oportunidades de socialização (festas, piqueniques, passeios, etc.) $(\beta=0,292, p<0,000)$

- É proporcionada formação suficiente e adequada para as funções que desempenha $(\beta=0,248, p<0,001)$ 
São fomentadas oportunidades de socialização (festas, piqueniques, passeios, etc.) $\left(R^{2}=0,402\right)$

- Considera útil e justo o Sistema de Avaliação de Desempenho $(\beta=0,255, p<0,000)$

- Participação nas decisões em especial nas que o afetam $(\beta=0,202, p<0,008)$

- Grau de Comunicação Interna - (os estatutos, regimentos e manuais de atividades, estão bem definidos e conhecidos por todos e os eventos divulgados atempadamente) $(\beta=0,159, p<0,0036)$

\section{Acha o seu trabalho não repetitivo $\left(\mathrm{R}^{2}=0,113\right)$}

- Grau de interatividade entre o IPG e a Comunidade em geral $(\beta=-0,254, p<0,002)$

- Participação nas decisões em especial nas que o afetam $(\beta=0,180, p<0,0023)$

Para testar as hipóteses, utilizou-se o teste $U$ de Mann-Whitney e o teste não-paramétrico de Kruskal-Wallis, seguido de comparações múltiplas das médias de ordem com a ANOVA Tukey. Apresentam-se os itens onde foram encontradas diferenças estatisticamente significativas relativamente a cada um dos fatores.

Gênero: Os trabalhadores do sexo masculino consideraram que têm mais oportunidades de socialização do que as mulheres (Tabela 8).

Tabela 8 - Teste U de Whitney para o Género

\begin{tabular}{ccccrr}
\hline \multirow{2}{*}{ Itens } & \multicolumn{4}{c}{ Género } \\
\cline { 2 - 5 } & Média & U & W & P \\
\hline $\begin{array}{l}\text { São fomentadas oportunidades de socialização } \\
\text { (festas, piqueniques, passeios, etc.) }\end{array}$ & 2,730 & 2794,000 & 7547,000 & 0,009 & $\begin{array}{r}\text { Homem> } \\
\text { Mulher }\end{array}$ \\
\hline
\end{tabular}

Atividade: Os trabalhadores docentes consideram que: os processos são muito burocratizados, são mais bem remunerados, recebem formação mais adequada, o sistema de avaliação de desempenho é mais útil e justo, têm mais oportunidades de socialização e estão mais satisfeitos com as condições físicas do trabalho do que os trabalhadores não docentes (Tabela 9).

Tabela 9 - Teste U de Whitney U para a Atividade

\begin{tabular}{|c|c|c|c|c|c|}
\hline \multirow{2}{*}{ Itens } & \multicolumn{4}{|c|}{ Atividade } & \\
\hline & Média & $U$ & W & $p$ & \\
\hline Os processos são fluidos (pouca papelada) & 2,320 & 2660,500 & 6840,500 & 0,002 & $\begin{array}{r}\text { Não } \\
\text { Docente> } \\
\text { Docente }\end{array}$ \\
\hline $\begin{array}{l}\text { A remuneração é adequada com as funções } \\
\text { desempenhadas }\end{array}$ & 2,820 & 1973,500 & 5213,500 & 0,000 & $\begin{array}{r}\text { Docente> } \\
\text { Não } \\
\text { Docente }\end{array}$ \\
\hline $\begin{array}{l}\text { É proporcionada formação suficiente e adequada } \\
\text { para as funções que desempenha }\end{array}$ & 3,050 & 2892,000 & 6132,000 & 0,016 & $\begin{array}{r}\text { Docente> } \\
\text { Não } \\
\text { Docente }\end{array}$ \\
\hline $\begin{array}{l}\text { Considera útil e justo o Sistema de Avaliação de } \\
\text { Desempenho }\end{array}$ & 2,330 & 2665,500 & 5905,500 & 0,002 & $\begin{array}{r}\text { Docente> } \\
\text { Não } \\
\text { Docente }\end{array}$ \\
\hline $\begin{array}{l}\text { São fomentadas oportunidades de socialização } \\
\text { (festas, piqueniques, passeios, etc.) }\end{array}$ & 2,730 & 2911,000 & 6151,000 & 0,018 & $\begin{array}{r}\text { Docente> } \\
\text { Não } \\
\text { Docente }\end{array}$ \\
\hline
\end{tabular}


Idade: $\mathrm{A}$ idade $(x 2=8,691$ e $\mathrm{p}=0,034)$ tem um efeito estatístico significativo $(\mathrm{p}<0,05) \operatorname{com} \mathrm{A}$ distribuição do trabalho é feita de forma justa (equitativa). Os trabalhadores com idade entre os 31-40 anos têm uma menor perceção de que a distribuição do trabalho seja feita de forma equitativa (média $=2,27$ ) relativamente à perceção dos trabalhadores com mais de 60 anos (média $=3,50)(p=0,044)$ (Tabela 10).

Tabela 10 - Teste de Kruskal-Wallis para a Idade

\begin{tabular}{cccc}
\hline \multirow{2}{*}{ Itens } & \multicolumn{3}{c}{ Idade } \\
\cline { 2 - 4 } & Média & $\chi \mathbf{2}$ & $\mathbf{p}$ \\
\hline A distribuição do trabalho é feito de forma justa (equitativa) & 3,050 & 8,691 & 0,034 \\
\hline
\end{tabular}

Unidade Orgânica: A Unidade Orgânica ( $x$ 2=16,698 e p=0,005) tem um efeito estatístico significativo $(p<0,05)$ com $A$ remuneração é adequada com as funções desempenhadas. 0 teste de Tukey destaca essa diferença. Os trabalhadores dos SC estão menos satisfeitos com a remuneração do que os trabalhadores da $\operatorname{ESTG}(p=0,014)$, da ESECD $(p=0,045)$ e da ESTH $(p=0,032)$. A ESTG, ESECD, ESTH classificaram este item como amarelo e as outras unidades orgânicas como vermelho.

A Unidade Orgânica ( $\chi 2=16,377$ e $p=0,006)$ tem um efeito estatístico significativo $(p<0,05)$ com as oportunidades de socialização. Os trabalhadores dos SC (média=2,27) consideraram ter menos oportunidades de socialização do que a ESS (média=3,5). A ESS é a única unida orgânica que classificou este item como amarelo. Todas as outras unidades orgânicas classificaram este item como vermelho.

A Unidade Orgânica ( $x 2=25,082$ e $p=0,000)$ tem um efeito estatístico significativo $(p<0,05)$ com Satisfação com as condições físicas de seu trabalho. O teste de Tukey destaca essa diferença. Os trabalhadores da ESS consideraram que as condições físicas do seu trabalho são piores que as da ESTG $(p=0,005)$ e do que das da ESTH $(p=0,002)$. A média deste item para a ESS é 2,75 correspondendo a uma classificação vermelha. Todas as outras unidades orgânicas tiveram uma classificação de amarela (Tabela 11).

Tabela 11 - Teste de Kruskal-Wallis para a Unidade Orgânica

\begin{tabular}{lccc}
\hline \multicolumn{1}{c}{ Items } & \multicolumn{3}{c}{ Unidade Orgânica } \\
\cline { 2 - 4 } & Media & x2 & $\mathbf{p}$ \\
\hline A remuneração é adequada com as funções desempenhadas & 2,820 & 16,698 & 0,005 \\
\hline $\begin{array}{l}\text { São fomentadas oportunidades de socialização (festas, piqueniques, } \\
\text { passeios, etc.) }\end{array}$ & 2,730 & 16,327 & 0,006 \\
\hline $\begin{array}{l}\text { As condições físicas do seu trabalho são satisfatórias (Ruído, Higiene, } \\
\text { Mobiliário, Equipamentos, Gabinetes) para desenvolver as suas atividades }\end{array}$ & 3,330 & 25,082 & 0,000 \\
\hline
\end{tabular}

Escolaridade: A Escolaridade ( $x 2=16,881$ e $p=0,010$ ) teve um efeito estatístico significativo $(p<0,05)$ com Os processos são fluidos. O teste de Tukey $(p=0,029)$ destaca essa diferença. Os docentes doutorados são os que consideraram os processos mais burocratizados (muita papelada), muito embora todas as classes de escolaridade classificaram os processos a vermelho.

A Escolaridade $(x 2=30,493$ e $p=0,000)$ teve efeito estatístico significativo $(p<0,05)$ com a adequação da remuneração. O teste de Tukey destaca essa diferença. Os trabalhadores com 
o doutoramento consideraram-se mais satisfeitos com a remuneração do que os dos 3 Ciclo $(p=0,000)$, do que os do Secundário $(p=0,002)$ e do que os trabalhadores com o Bacharelato $(p=0,021)$. Além disso, os Mestres estão mais satisfeitos com a remuneração do que os trabalhadores do 3 iclo. Somente os trabalhadores com o doutoramento, com uma média igual a 3,34, tiveram uma classificação de amarelo. Todos os outros grupos tiveram uma classificação vermelha.

A Escolaridade ( $x 2=15,114$ e $p=0,019)$ teve um efeito estatístico significativo $(p<0,05)$ com o Sistema de Avaliação de Desempenho. O teste de Tukey $(p=0,019)$ destaca essa diferença. Os licenciados consideraram o Sistema de Avaliação de Desempenho menos útil e menos justo do que os trabalhadores com doutoramento $(p=0,019)$, apesar de todas as classes de escolaridade terem classificado este item como vermelho (Tabela 12).

Tabela 12 - Teste de Kruskal-Wallis para a Escolaridade

\begin{tabular}{lccc}
\hline \multicolumn{1}{c}{ Itens } & \multicolumn{3}{c}{ Escolaridade } \\
\cline { 2 - 4 } & Média & $\mathbf{x}$ & $\mathbf{p}$ \\
\hline Os processos são fluidos (pouca papelada) & 2,320 & 16,881 & 0,010 \\
\hline A remuneração é adequada com as funções desempenhadas & 2,820 & 30,493 & 0,000 \\
\hline Considera útil e justo o Sistema de Avaliação de Desempenho & 2,330 & 15,181 & 0,019 \\
\hline
\end{tabular}

Não foram encontradas diferenças significativas relativamente ao Tempo de serviço no IPG ( Tenure) para nenhum dos 31 itens.

Categoria Profissional: A Categoria Profissional ( $x 2=19,614$ e $p=0,012)$ teve um efeito estatístico significativo $(p<0,05)$ com o item Os processos são fluidos. O teste de Tukey $(p=0,028)$ destaca essa diferença. Os Professores Adjuntos ou Equivalentes consideram os processos mais burocratizados do que os Assistentes Operacionais.

A Categoria Profissional ( $x 2=46,725$ e $p=0,000$ ) teve um efeito estatístico significativo $(\mathrm{p}<0,05)$ com $A$ Remuneração é adequada. O teste de Tukey destaca essa diferença. $O$ Professor Adjunto ou Equivalente classificou mais favorável a remuneração do que o Assistente $(p=0,010)$, do que o Técnico Superior $(p=0,001)$, do que o Assistente Técnico $(p=0,000)$ e do que $o$ Assistente Operacional $(p=0,000)$.

A Categoria Profissional $(x 2=21,700$ e $p=0,006)$ teve um efeito estatístico significativo $(p<0,05)$ com o Sistema de Avaliação de Desempenho. O teste de Tukey $(p=0,006)$ destaca essa diferença. O Professor Adjunto ou Equivalente considerou o Sistema de Avaliação de Desempenho mais útil e justo do que o Técnico Superior (Tabela 13).

Tabela 13 - Teste de Kruskal-Wallis para a Categoria Profissional

\begin{tabular}{lccc}
\hline \multirow{2}{*}{ Itens } & \multicolumn{3}{c}{ Categoria Profissional } \\
\cline { 2 - 4 } & Média & $\mathbf{2}$ & $\mathbf{p}$ \\
\hline Os processos são fluidos (pouca papelada) & 2,320 & 19,614 & 0,012 \\
\hline A remuneração é adequada com as funções desempenhadas & 2,820 & 46,725 & 0,000 \\
\hline Considera útil e justo o Sistema de Avaliação de Desempenho & 2,330 & 21,700 & 0,006 \\
\hline
\end{tabular}

Não foram encontradas diferenças estatisticamente significativas na satisfação, conforme determinado pela one-way ANOVA para o clima organizacional ou para as dimensões em 

Profissional.

O estudo do clima organizacional constitui o primeiro passo para desenvolver estratégias segmentadas e customizadas, que vão ao encontro das reais necessidades dos trabalhadores e também procurar melhorar o desempenho das instituições. Nesse sentido deixam-se algumas, modestas, sugestões e recomendações à liderança do IPG suportadas nos trabalhos de Robin e Judge (2019) e Greenberg (2011b).

\section{Sugestões e Recomendações}

Embora a classificação geral do Clima Organizacional no IPG seja ligeiramente positiva, ainda existe espaço para melhoramento. De acordo com os resultados obtidos propõe-se:

- Oportunidades de Socialização - desenvolver atividades de socialização em todas as unidades orgânicas, muito em especial nos Serviços Centrais. Festas, piqueniques, caminhadas, campanhas de qualidade de vida, palestras focadas no bem-estar e alimentação saudável, que estimulem práticas de exercícios físicos e alertem para os perigos de hábitos nocivos como o fumo e o alcoolismo, são boas maneiras de incentivar os trabalhadores e melhorar a sua satisfação;

- Melhoria das Condições de Trabalho - em especial as condições de trabalho dos trabalhadores da Escola Superior de Saúde. Equipar estes trabalhadores com os meios necessários para realizarem as suas atividades num ambiente seguro e livre de ameaças de perigo físico ou psicológico. As condições físicas das instalações (higiene, design do local de trabalho, etc.) interferem no grau de satisfação dos trabalhadores;

- Empoderamento - descentralização do poder para permitir maior participação dos trabalhadores no processo de tomada de decisão. Dar aos trabalhadores a oportunidade de participar no planeamento dos objetivos do IPG, especialmente na Escola Superior de Tecnologia e Gestão, no geral para os trabalhadores docentes e, em particular, para os docentes Assistentes ou equiparados (média=2,38);

- Redesenho dos Processos - os processos inerentes com a Gestão da Qualidade precisam de ser redesenhados (revistos) para se tornarem menos burocratizados especialmente os relacionados com os trabalhadores docentes e, em particular, os doutores (média $=1,98$ ) e mestres (média $=2,00$ ) que consideram os processos pouco fluidos (muita papelada);

- Expectativas de Promoção - criar nos trabalhadores a sensação de que o seu esforço resultará num determinado desempenho com a consequente recompensa que valorizem por exemplo, a possibilidade de progressão na carreira, reconhecimento do trabalho desenvolvido nos canais de comunicação do Instituto Politécnico da Guarda (por exemplo, na rádio do IPG), diplomas, medalhas, etc. Atenção especial deve ser dada aos trabalhadores não docentes ( $m e ́ d i a=2,39$ );

- Sistema de Avaliação de Desempenho - dar formação aos avaliadores e esclarecer da equidade e importância do Sistema de Avaliação de Desempenho para o crescimento profissional do trabalhador em todas as unidades orgânicas, mas, principalmente nos Serviços Centrais (média=1,91);

- Adequação da Remuneração - divulgar e esclarecer que o processo de remuneração depende da lei geral. Os docentes estão mais satisfeitos com a remuneração (média $=3,23$ ) do que os trabalhadores não docentes ( média = 2,35). 


\section{CONCLUSÕES}

O clima organizacional repercute-se no desempenho de qualquer instituição pública ou privada. Um clima organizacional positivo incentiva e sustenta a motivação dos trabalhadores. A motivação dos trabalhadores é influenciada por muitos fatores, incluindo o clima organizacional, e os seus efeitos refletem-se na produtividade. É, portanto, fundamental avaliar e analisar o clima organizacional de qualquer instituição. Tendo por base esta preocupação, esta pesquisa visou avaliar, compreender e melhorar o clima organizacional do Instituto Politécnico da Guarda (IPG) que luta para melhorar a sua imagem a fim de atrair novos alunos tanto nacionais como internacionais, aumentar a interação com a comunidade e melhorar e ampliar a investigação que desenvolve.

Embora os resultados mostrem que o clima organizacional no IPG é ligeiramente positivo (média =3.29), ainda existe uma grande oportunidade de melhoria. A Escola Superior de Turismo e Hotelaria destaca-se das demais pela positiva. A Escola Superior de Tecnologia e Gestão regista o menor valor médio de satisfação com o clima organizacional. Em média, os trabalhadores docentes estão mais satisfeitos com o clima organizacional do que os trabalhadores não docentes.

Os trabalhadores de todas as unidades orgânicas gostariam de participar no planeamento dos objetivos do IPG, em especial os trabalhadores docentes da Escola Superior de Tecnologia e Gestão. Todos os trabalhadores, mas especialmente os trabalhadores não docentes, afirmaram que o Sistema de Avaliação de Desempenho não é justo e útil. Esse sentimento é ainda mais forte nos Serviços Centrais. Também os trabalhadores, em todas as unidades orgânicas, gostariam de ter mais oportunidades de socialização, mas essa incidência tem mais pertinência nos Serviços Centrais.

Os docentes consideram os processos demasiado burocratizados, especialmente os doutores e os mestres. Somente os trabalhadores da Escola Superior de Saúde consideraram que as condições físicas do seu trabalho são não satisfatórias.

No geral, todos os trabalhadores consideraram que a remuneração não é adequada com as funções desempenhadas com forte ênfase para os trabalhadores não docentes. Além disso, todos eles têm poucas expectativas de virem a ser promovidos.

Em suma, o clima organizacional no IPG teve um impacto diferenciado na satisfação dos trabalhadores o que implica a aplicação de medidas de melhoria distintas.

Em termos de implicações práticas, este estudo contribui para uma melhor compreensão das variáveis e dimensões que podem influenciar o clima organizacional nas unidades orgânicas das Instituições de Ensino Superior. É necessário promover o estudo e a compreensão do clima organizacional de qualquer instituição para atingir níveis de excelência e alto desempenho, bem como encontrar soluções que aumentem tanto a satisfação como a motivação dos trabalhadores com o consequente aumento do nível de desempenho.

Em termos de limitações, este estudo trata exclusivamente de uma Instituição de Ensino Superior. Assim, as peculiaridades inerentes a esta instituição podem limitar a generalização dos resultados do estudo para todas as Instituições de Ensino Superior. Como indicação para futuras investigações sugerimos a aplicação deste estudo a outras Instituições de Ensino Superior bem como reavaliar e comparar temporalmente o clima organizacional do IPG no médio prazo. 


\section{REFERÊNCIAS}

Aarabi, M.S., Subramaniam I. D., \& Akeel A. B. (2013). Relationship between Motivational Factors and Job Performance of Employees in Malaysian Service Industry. Canadian Center of Science and Education, Asian Social Science, 9(9): 301-308.

Abdelhadi, N., \& Drach-Zahavy, A. (2012). Promoting patient care: Work engagement as a mediator between ward service climate and patient-centered care. Journal of Advanced Nursing, 68(6), $1276-$ 1287.

Andersen, L.B., Heinesen, E. and Pedersen, L.H. (2016). Individual performance: from common source bias to institutionalized assessment. Journal of Public Administration Research and Theory, Vol. 26 No. 1, pp. 63-78. [ISI].

Armstrong, M. (2012). A Handbook of Human Resource Management Practice. London: Kogan.

Asim, M. (2013). Impact of motivation on employee performance with the effect of training: specific to education sector of Pakistan. International Journal of Scientific and Research Publications, Volume 3, pp. 1-9.

Bennis, W. G. \& Schein, E. H. (1966). Personal and Organizational Change Trough Group Methods. The Laboratory Approach.

Brown, D. R. ( 2011). An Experiential Approach to Organizational Development(8th ed.). New Jersey. Person Education, Inc.

Brown, S. P., \& Lam, S. K. (2008). A meta-analysis of relationships linking employee satisfaction to customer responses. Journal of Retailing, 84, 243-255.

Chen, S., \& Liu, P. (2012). Effects of internal marketing, organizational commitment, job involvement and job satisfaction on work performance: A study of the elderly care institutions in Taiwan. Marketing Review, 9(3), 277-302.

Chintalloo, S., \& Mahadeo, J. (2013). Effect of Motivation on Employees' Work Performance at Ireland Blyth Limited: Proceedings of 8th Annual London Business Research Conference Imperial College, London, UK, 8.

Colquitt, J. A., LePine, J. A., \& Wesson, M. J. (2015). Organizational Behavior: Improving Performance and Commitment in the Workplace. New York: McGraw-Hill Companies.

Corsini, R. J. (1999). The dictionary of psychology. Philadelphia, PA: Taylor \& Francis.

Edwards, B. D., \& Bell, S. T. (2008). Relationships between facets of job satisfaction and task and contextual performance. Applied Psychology: An International Review, 57(3), 441-465.

Ehrhart, M. G., \& Raver, J. L. (2014). The effects of organizational climate and culture on productive and counterproductive behavior. In B. Schneider \& K. Barbera (Eds.), The Oxford handbook of organizational climate and culture (pp. 153-176). New York: Oxford University Press.

Ehrhart, M. G., Schneider, B., \& Macey, W. H. (2014). Organizational climate and culture: An introduction to theory, research, and practice. New Y1ork: Routledge.

Fu, W., Deshpande, S. P., \& Zhao, X. (2011). The impact of ethical behavior and facets of job satisfaction on organizational commitment of Chinese employees. Journal of Business Ethics, 104 , 537-543.

Galer, J. B., Vrieesendorp, S., \& Ellis, A. (2005). Managers Who Lead. Cambridge, Massachusetts: Management Sciences for Health.

George, J. M., \& Jones, G. R. (2012). Understanding and Managing Organizational Behavior. New Jersey: Pearson Prentice-Hall.

Goleman, D. (2000). Leadership the Gets Results. Harvard Business Review (March-April). 
Gong, Y., Law, C. S., \& Xing, K. S. R. (2009). Human resources management and firm performance: The differential role of managerial affective and continuance commitment. Journal of Applied Phycology, 94.

Greenberg, J. (2011a). Promote procedural and interactional justice to enhance individual I and organizational outcomes. In E. A. Locke (Eds). Blackwell handbook of principles of organizational behavior ( $2^{\text {nd }}$ ed.). Malden MA: Blackwell Publishers.

Greenberg, J. (2011b). Behavior in Organizations. Pearson Educated Limited. Prentice Hall. USA.

Greenslade, J. H., \& Jimmieson, N. L. (2011). Organizational factors impacting on patient satisfaction: A cross sectional examination of service climate and linkages to nurses' effort and performance. International Journal of Nursing Studies, 48(10), 1188-1198.

Hira, A., \& Waqas, I. (2012). A Study of job satisfaction and IT's Impact on the performance in the banking industry of Pakistan. International Journal of Business and Social Science, 3(19), 174-180.

Iqbal, M. T., Latif, W., \& Naseer, W. (2012). The impact of person job fit on job satisfaction and its subsequent impact on employee's performance. Mediterranean Journal of Social Sciences, 3(2), 523-530.

Ivancevich, J. M., Konopaske, R., \& Matteson. M. T. (2014). Organizational Behaviour and Management. New York: McGraw-Hill/Irwin.

Jiang, K., Chuang, C., \& Chiao, Y. (2015). Developing collective customer knowledge and service climate: The interaction between service-oriented high-performance work systems and service leadership. Journal of Applied Psychology, 100(4), 1089-1106.

Judge, T. A., \& Church, A. H. (2000). Job satisfaction: Research and Practice. In C. A. Cooper \& E. A. Locke (Eds). Industrial and organizational psychology: linking theory to practice. Malden, MA: Blackwell.

Keller, E., \& Aguiar, M. A. F. (2004). Análise Critica Teórica da Evolução do Conceito de Clima Organizacional. Terra e Cultura, 20, 91-113.

Landy, F. J. \& Conte, J. M. (2013). Work in The 21st Century: An Introduction to Industrial and Organizational Psychology. Mason: John Wiley \& Sons.

Limm, H., Guendel, H., Heinmueller, M., Marten-Mittag, B., Nater, U. M., \& Siegrist, J. (2011). Stress management interventions in the workplace improve stress reactivity: a randomized controlled trial. Occupational and Environmental Medicine, 68, 126-133.

Locke, E. A. (1976). The nature and causes of job satisfaction. In M. D. Dunnette (Ed.), Handbook of industrial and organizational psychology (pp. 1297-1349). Chicago, IL: Rand McNally.

Malik, M. E., Danish, R., \& Usman, A. (2010a). Impact of job climate and extrinsic rewards on job satisfaction of banking executives: A case of Pakistan. Interdisciplinary Journal of Contemporary Research in Business, 12, 125-139.

Malik, M. E., Nawab, S., Naeem, B., \& Danish, R. Q. (2010b). Job satisfaction and organizational commitment of university teachers in Public sector of Pakistan. International Journal of Business and Management, 5(6), 17-26.

Mallery, P., \& George, D. (2016). IBM SPSS Statistics 23 Step by Step: A Simple Guide and Reference.14th Edition. New York, Routledge.

Maroco, J. (2014). Análise Estatística - Com utilização do SPSS. Lisboa: Edições Silabo, Lda-Lisboa.

Mitonga-Monga, J., Flotman, A. P., \& Cilliers, F. (2018). The relationship between job satisfaction and organizational citizenship behavior: a Democratic Republic of Congo organizational perspective. Acta Commercii - Independent Research Journal in the Management Science, 1-8.

Muchinsky, P. M. (2003). Psychology applied to work. Belmont, CA: Thomson Wadsworth.

Murphy, S. (2015). The optimistic workplace: Creating an environment that energizes everyone. New York: AMACON. 
Neal, A., \& Griffin, M. A. (2006). A study of the lagged relationships among safety climate, safety motivation, safety behavior, and accidents at the individual and group levels. Journal of Applied Psychology, 91, 946-953.

Noblet, A., \& La Montagne, A. D. (2006). The role of workplace health promotion in addressing job stress. Health Promotion International, 21, 346-353.

Ostroff, C., Kinicki, A. J., \& Muhammad, R. S. (2012). Organizational culture and climate. In N. W. Schmitt \& S. Highhouse (Eds.), Handbook of psychology, Vol. 12: Industrial and lorganizational psychology (2d ed., pp. 643-676). Hoboken, NJ: Wiley.

Owus, T. (2012). Effects of Motivation on Employee Performance: A Case Study of Ghana Commercial Bank, Kumasi Zone. Master's Thesis. Institute of Distance Learning, KNUST.

Passwan, A, \& Ganesh, G. (2009). Higher education institutes: satisfaction and loyalty among. Journal of Marketing for Higher Education, 19(1), 65-84.

Patterson, M. G., West, M. A., Shackleton, V. J., Dawson, J. F., Lawthom, R., Maitlis, S., \& Wallace, A. M. (2005). Validating the organizational climate measure: Links to managerial practices, productivity and innovation. Journal of Organizational Behavior, 26, 379-408.

Qureshi, J. A., Hayat, K., Ali, M., \& Sarwat, N. (2012). Impact of job satisfaction and organizational commitment on employee performance, evidence from Pakistan. Interdisciplinary Journal of Contemporary Research in Business, 3(4), 642-657.

Ritz, A., Brewer, G.A. and Neumann, O. (2016). Public service motivation: a systematic literature review and outlook". Public Administration Review, Vol. 76 No. 3, pp. 414-426.

Robins, S. P., \& Judge, T. A. (2019). Organizational Behavior. Pearson Education, $18^{\text {th }}$ Edition. Prentice-Hall - USA..

Samad, S., \& Selangor, S. A. (2005). Unraveling the organizational commitment and job performance relationship: exploring the moderating effect of job satisfaction. The Business Review, 4(2), 79-84.

Schneider, B. \& Reichers, A. E. (1990). Climate and culture: An evolution of constructs. In B. Schneider (Ed.), Organizational climate and culture (pp. 129-150). San Francisco, NC: The Jossey-Bass.

Schneider, B., Ehrhart, M. G., \& Macey, W. H. (2013). Organizational climate and culture. Annual review of psychology, 64 (361-388).

Schuler, R. S. \& Jackson, S. E. (2007). Strategic Human Resource management (second edition). Blackwell Publishing Ltd.

Shahzad, I., Javad, P., Pirzada, S., Nasreen, S., \& Khanam, F. (2014). Impact of Employee Motivation on Employee Performance. European Journal of Business and Management, 6(23): 159-166.

Smith, V. (2013). Sociology of Work: An Encyclopedia. California: SAGE Publications.

Springer, G. J. (2011). A study of job motivation, satisfaction, and performance among bank employees. Journal of Global Business Issues, 5(1), 29-42.

Tamayo, A. (2001). Prioridades Axiológicas, Atividade Física e Estresse Ocupacional. Revista de Administração Contemporânea, 5, 127-147.

Vogel, R., Homberg, F., \& Gericke, A. (2016). Abusive supervision, public service motivation, and employee deviance: the moderating role of employment sector. Evidence-based HRM: A Global Forum for Empirical Scholarship, Vol. 4 No. 3, pp. 214-231.

Whitman, D. S., Van Rooy, D. L., \& Viswesvaran, C. (2010). Satisfaction, citizenship behaviors and performance in work units: A meta-analysis of collective construct relations. Personnel Psychology, $63,41-81$.

Wright, T. A., Cropanzano, R., \& Bonnet D. G. (2007). The moderating role of employee wellbeing on the relationship between job satisfaction and job performance. Journal of Occupational Health Psychology (12(2): 93-104. 